\title{
Substance Use Among Middle-Aged and Older Lesbian, Gay, and Bisexual Adults in the United States, 2015 to 2017
}

\author{
Benjamin H. Han, MD ${ }^{1,2,3} \oplus^{0}$, Mari Miyoshi, BA ${ }^{4}$, and Joseph J. Palamar, PhD 2,3 \\ 'Department of Medicine, Division of Geriatric Medicine and Palliative Care, New York University School of Medicine, New York, NY, USA; \\ ${ }^{2}$ Department of Population Health, New York University School of Medicine, New York, NY, USA; ${ }^{3}$ Center for Drug Use and HIV/HCV Research, New \\ York University School of Global Public Health, New York, NY, USA; ${ }^{4} J$ John A. Burns School of Medicine, University of Hawaii, Honolulu, HI, USA.
}

J Gen Intern Med 35(12):3740-41

DOI: $10.1007 / \mathrm{s} 11606-020-05635-2$

(c) Society of General Internal Medicine 2020

\section{INTRODUCTION}

Research has shown that lesbian, gay, and bisexual (LGB) young adults have substantially higher rates of substance use compared with heterosexuals. ${ }^{1}$ Many have attributed these higher rates to minority stressors including discrimination, concealment, oppression, and stigma. ${ }^{2}$ Few studies, however, have focused on substance use among older LGB adults. Older adults are more likely to have chronic medical disease and take prescription medications that can interact with substance use and increase their risk for adverse events. Older LGB adults may also have additional stressors including social isolation and stigma around aging which may increase substance use risk. The objective for this study was to use cross-sectional data from a nationally representative sample of adults in the United States to estimate the prevalence of substance use among middle-aged and older LGB adults and examine their risk of use compared with heterosexuals.

\section{METHODS}

We examined aggregated data from respondents age $\geq 50$ from the 2015 to 2017 National Survey on Drug Use and Health, ${ }^{3}$ an annual cross-sectional survey of a nationally representative sample of non-institutionalized individuals in the United States. We estimated and compared prevalence of past-year use of cannabis (any use and non-medical use), alcohol, cocaine, methamphetamine, and misuse of prescription opioids, sedatives, stimulants, and tranquilizers between adults identifying as LGB and heterosexual. Prescription misuse was defined as using in any way not directed by a doctor, including use without a prescription, use in greater amounts, more often, or longer than instructed to take them, or use in any other way a doctor did not direct. ${ }^{3}$ We also estimated and compared the prevalence of alcohol use disorder ${ }^{4}$ and nicotine dependence ${ }^{5}$

Received November 12, 2019

Revised December 4, 2019

Accepted January 3, 2020

Published online June 1, 2020 between the two groups. Comparisons of prevalence estimates were conducted using chi-square. We then calculated adjusted odds ratios (aORs) for LGB adults' odds of use relative to heterosexual adults, controlling for age, race, gender, marital status, and family income. We used weights (provided by NSDUH) to account for complex survey design, selection probability, non-response, and population distribution. We used imputation-revised variables when available to limit missing data.

\section{RESULTS}

The analytic sample included 25,880 participants with $2.5 \%$ identifying as a sexual minority (with $1.5 \%$ gay or lesbian and $1.0 \%$ bisexual). Table 1 presents prevalence estimates and logistic regression results for the 11 substance categories. LGB middle-aged and older adults had a significantly higher prevalence than heterosexuals for all substances examined with exception of nicotine dependence. In adjusted analyses, LGB adults age $\geq 50$ were at higher odds of reporting pastyear non-medical cannabis use $(\mathrm{aOR}=1.96,95 \%$ confidence interval $[\mathrm{CI}]=1.36,2.83)$, alcohol use $(\mathrm{aOR}=1.33,95 \% \mathrm{CI}$ $1.06,1.66)$, prescription opioid misuse $(\mathrm{aOR}=1.66,95 \% \mathrm{CI}$ $1.09,2.53)$, and prescription tranquilizer misuse $(\mathrm{aOR}=2.39$, $95 \%$ CI 1.31, 4.34) compared with heterosexuals.

\section{DISCUSSION}

Several studies show that rates of substance use are higher among LGB persons compared with those among heterosexuals, particularly among adolescents and young adults. However, to our knowledge, our national estimates are the first to focus on LGB adults age $\geq 50$. Our findings confirm that higher prevalence of substance use among LGB adults continues into later life, which may be related to the chronic stressors of aging and LGB discrimination. This has important and unique health implications as physiological changes of aging along with increases in chronic medical diseases and prescribed medications, place older adults at increased vulnerability to the harms of substance use.

Our analyses found a higher adjusted odds ratio of cannabis use and prescription misuse of opioids and tranquilizers 
Table 1 Past-Year Substance Use Prevalence Among Middle-aged and Older Adults by Sexual Orientation in the United States, 2015-2017

\begin{tabular}{|c|c|c|c|c|}
\hline & $\begin{array}{l}\text { Heterosexual adults ages } \geq \mathbf{5 0} \\
(n=25,217), \text { weighted } \% \\
(95 \% \text { CI })\end{array}$ & $\begin{array}{l}\text { LGB adults ages } \geq 50 \\
(n=663), \text { weighted } \%(95 \% \text { CI })\end{array}$ & $X^{2} p$ value & aOR $(95 \%$ CI)* \\
\hline Cannabis use (any) & $6.46(6.07,6.87)$ & $17.70(13.60,22.80)$ & $<0.001$ & $2.21(1.58,3.08)$ \\
\hline Non-medical cannabis use & $5.45(5.13,5.79)$ & $13.90(10.20,18.70)$ & $<0.001$ & $1.96(1.36,2.83)$ \\
\hline Alcohol use & $62.60(61.7,63.5)$ & $70.60(66.30,74.60)$ & 0.001 & $1.33(1.06,1.66)$ \\
\hline Alcohol use disorder ${ }^{\dagger}$ & $3.36(3.10,3.64)$ & $5.29(3.62,7.67)$ & 0.02 & $1.20(0.78,1.84)$ \\
\hline Nicotine dependence & $6.48(6.18,6.80)$ & $8.16(5.98,11.0)$ & 0.15 & $0.97(0.68,1.38)$ \\
\hline Cocaine use & $0.65(0.54,0.79)$ & $2.33(1.21,4.42)$ & $<0.001$ & $1.86(0.88,3.89)$ \\
\hline Methamphetamine use & $0.30(0.24,0.37)$ & $0.79(0.31,2.04)$ & 0.05 & $1.75(0.63,4.87)$ \\
\hline Prescription opioid misuse & $2.34(2.15,2.55)$ & $4.72(3.19,6.94)$ & 0.001 & $1.66(1.09,2.53)$ \\
\hline Prescription sedative misuse & $0.49(0.39,0.61)$ & $0.93(0.50,1.69)$ & 0.05 & $1.31(0.65,2.65)$ \\
\hline Prescription tranquilizer misuse & $1.07(0.92,1.25)$ & $3.62(2.11,6.17)$ & $<0.001$ & $2.39(1.31,4.34)$ \\
\hline Prescription stimulant misuse & $0.27(0.22,0.34)$ & $0.76(0.30,1.91)$ & 0.02 & $1.85(0.68,5.02)$ \\
\hline
\end{tabular}

*Adjusted for age, gender, race, family income, and marital status. ${ }^{\dagger}$ Based on the Diagnostic and Statistical Manual of Mental Disorders, Fourth Edition. ${ }^{4}$ Based on Nicotine Dependence Syndrome Scale. ${ }^{5}$ LGB, lesbian, gay, or bisexual; aOR adjusted odds ratio

among older LGB adults. Cannabis use by LGB adults has been shown to be higher for adults ages $\geq 18$ compared with that for heterosexuals, ${ }^{2}$ and our results confirm these differences among older adults. Misuse of prescription opioids and tranquilizers (such as benzodiazepines) is particularly concerning as it not only represents a highly risky behavior for older adults, but also highlights the possible undertreatment of pain or mental health distress. ${ }^{6}$ Substance use adds complexity to caring for older adults, but especially so for underserved vulnerable populations such as older LGB adults who experience a range of health disparities and have barriers to accessing healthcare. ${ }^{2}$ Prevention and interventions for unhealthy substance use should focus on older LGB populations to reduce harms and deliver culturally competent, patient-centered approaches to care.

Corresponding Author: Benjamin H. Han, MD; Department of Medicine, Division of Geriatric Medicine and Palliative Care New York University School of Medicine, New York, NY, USA (e-mail: Benjamin. Han@nyumc.org).

This work has not been presented before.

Funding Information This research was funded by the following grants through the National Institutes of Health: K23DA043651 (Han) and K01DA038800 from the National Institute on Drug Abuse (Palamar), T35AG050998-01 from the National Institute on Aging (Miyoshi). The National Institutes of Health provided financial support for the project and the preparation of the manuscript but did not have a role in the design of the study, the analysis of the data, the writing of the manuscript, nor the decision to submit the present research.

\section{Compliance with Ethical Standards:}

Conflict of Interest: The authors declare that they do not have a conflict of interest.

Disclaimer: The content is solely the responsibility of the authors and does not necessarily represent the official views of the National Institutes of Health.

Publisher's Note: Springer Nature remains neutral with regard to jurisdictional claims in published maps and institutional affiliations.

\section{REFERENCES}

1. Medley G, Lipari RN, Bose J, Cribb DS, Kroutil LA, McHenry G. Sexual orientation and estimates of adult substance use and mental health: Results from the 2015 National Survey on Drug Use and Health. NSDUH Data Review. Available at: https://www.samhsa.gov/data/sites/default/ files/NSDUH-SexualOrientation-2015/NSDUH-SexualOrientation-2015/ NSDUH-SexualOrientation-2015.htm Accessed 31 Oct 2019.

2. Cahill SR. Research and Policy Change to Improve Healthcare and Elder Services for LGBT Older Adults. LGBT Health 2017;4:381-383.

3. Center for Behavioral Health Statistics and Quality. Reports and Detailed Tables From the 2017 National Survey on Drug Use and Health (NSDUH). Available at: https://www.samhsa.gov/data/nsduh/reports-detailed-tables-2017-NSDUH. Accessed 31 Oct 2019.

4. American Psychiatric Association. Diagnostic and Statistical Manual of Mental Disorders. 4th ed. Washington, DC: American Psychiatric Association; 1994.

5. Shiffman S, Waters A, Hickcox $\mathbf{M}$. The nicotine dependence syndrome scale: a multidimensional measure of nicotine dependence. Nicotine Tob Res 2004; 6:327-348.

6. Fredriksen-Goldsen KI, Kim HJ, Shui C, Bryan AEB. Chronic Health Conditions and Key Health Indicators Among Lesbian, Gay, and Bisexual Older US Adults, 2013-2014. Am J Public Health. 2017;107:1332-1338.

Publisher's Note Springer Nature remains neutral with regard to jurisdictional claims in published maps and institutional affiliations. 